Article

\title{
Synthesis of New Naphtho[2,3-f]quinoxaline-2,7,12(1H)-trione and Anthra-9,10-quinone Dyes from Furan-2,3-diones
}

\section{Şevket Hakan Üngören}

Department of Chemistry, Faculty of Arts and Sciences, Bozok University, Yozgat, Turkey. Tel.: +90 354 2421022; fax: +90 354 2421022; E-mails: hungoren@erciyes.edu.tr; shakan.ungoren@bozok.edu.tr

Received: 11 March 2009; in revised form: 26 March 2009 / Accepted: 30 March 2009 / Published: 2 April 2009

\begin{abstract}
Novel naphtho[2,3-f]quinoxaline-2,7,12(1H)-trione and anthra-9,10-quinone dyes were synthesized in good yield from furan-2,3-diones using 1,2-diaminoanthra-9,10quinone and 1,4-diaminoanthra-9,10-quinone. The chromophores were characterized by molecular spectroscopy methods.
\end{abstract}

Keywords: Naphtho[2,3-f]quinoxaline-2,7,12(1H)-triones; Anthra-9,10-quinones; Furan2,3-diones; Diaminoanthra-9,10-quinone; Dye modification; Vat dyes.

\section{Introduction}

Anthra-9,10-quinones and their condensed derivatives with heterocycles such as indanthrone (Pigment Blue 60, I), anthrapyrimidine (Pigment Yellow 108, II) and Vat Yellow 3 (III) (Figure 1) possess brilliant hues and very good fastness and represent an important group of vat dyes for the textile industry $[1,2]$. In addition to these properties, some anthra-9,10-quinone dyes are widely used in other fields, such as in medicine and food chemistry [3] and high-technology systems [4]. Consequently, anthra-9,10-quinones are interesting compounds from the viewpoint of both their reactions and applications.

Furan-2,3-diones (1) have been shown to be very useful synthons for the preparation of various heterocycles. These compounds show typical carbonyl and lactone reactions, depending on the structures of the nucleophiles involved [5-8]. For example, furan-2,3-diones undergo cyclocondensations with 1,2-diamines to provide the corresponding quinoxalines and aromatic amines 
react with furan-2,3-diones to give the corresponding Schiff bases and pyrrole-2,3-dione derivatives, depending on the reaction times and temperature $[5,9]$.

Figure 1. Some commercial vat dyes.<smiles>O=C(Nc1ccccc1)c1ccc2c(c1)C(=O)c1ccc(C(=O)Nc3ccccc3)cc1C2=O</smiles>

Furan-2,3-diones (1) can offer many possibilities for the construction of various heterocyclic dyes including the new naphtho[2,3-f]quinoxaline and anthra-9,10-quinone derivatives reported herein. In this work we present new anthra-9,10-quinone (2) and naphtho[2,3-f]quinoxaline (3) dyes derived from the reactions of some furan-2,3-diones with 1,2-diaminoanthra-9,10-quinone (1,2-DAAQ) and 1,4-diaminoanthra-9,10-quinone (1,4-DAAQ), acting as bifunctional nucleophiles.

\section{Results and Discussion}

Furan-2,3-dione starting materials 1a-f were prepared according to the literature [10-13]. The $\mathrm{C}_{5}$ atom of compounds 1a-d smoothly reacted with the amino group of 1,2-DAAQ and 1,4-DAAQ to give compoubds 2 under mild conditions and in high yields (75-90\%, Scheme 1).

Scheme 1. Synthesis of compounds 2.

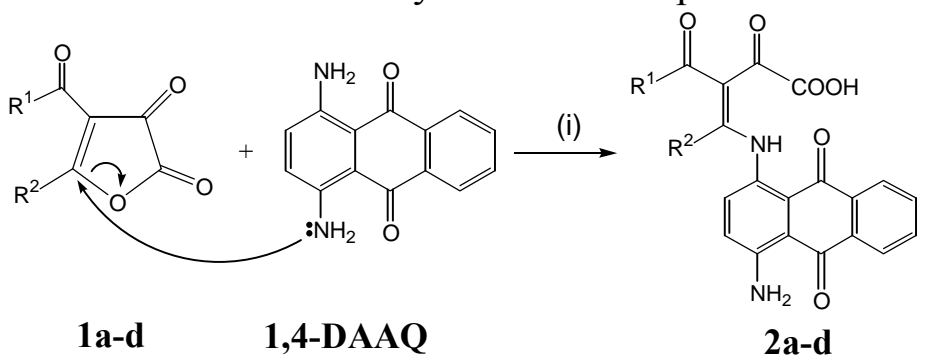

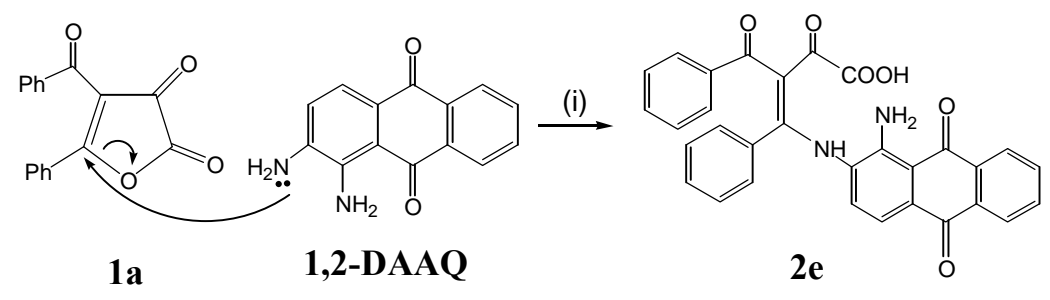

1, 2: $\mathbf{a} \mathrm{R}^{1}, \mathrm{R}^{2}=\mathrm{Ph} ; \mathbf{b} \mathrm{R}^{1}, \mathrm{R}^{2}=4-\mathrm{MeOPh} ; \mathrm{R}^{1}, \mathrm{R}^{2}=4-\mathrm{MePh} ; \mathbf{d} \mathrm{R}^{1}=\mathrm{Me}, \mathrm{R}^{2}=4-\mathrm{MePh}$

Reaction conditions: (i) Benzene, RT, $48 \mathrm{~h}$. 
Due to the greater reactivity of the amino group attached to the $C_{2}$ atom of 1,2-DAAQ, compared with the amino group attached to the $\mathrm{C}_{1}$ atom of 1,2-DAAQ, 1,2-DAAQ was modified from the amino group attached to $\mathrm{C}_{2}$-position of 1,2-DAAQ to give 2e. On the other hand, the amino group attached to the $\mathrm{C}_{2}$ atom of 1,2-DAAQ did not react with the $\mathrm{C}_{5}$ atom of $1 \mathrm{a}$ at higher temperature, but reacted with the $\mathrm{C}_{3}$ atom of $\mathbf{1 a}$ by forming a Schiff base, which was not isolated (as outlined in Scheme 2). Through attack of the second amino group on the lactone carbonyl group, ring opening occurs. The reactions of 1,2-DAAQ with 1b,c,e,f run via the same reaction pathways to give $\mathbf{3}$ in nearly quantitative yields of $90-96 \%$ in boiling benzene. This proposed mechanism is similar to that reported in the literature for the reaction pathways of furan-2,3-diones with 1,2-diamino nucleophiles [9,14].

Scheme 2. Synthesis of compounds 3.<smiles>[R]C(=O)C1=C([R])OC(=O)C1=O</smiles>

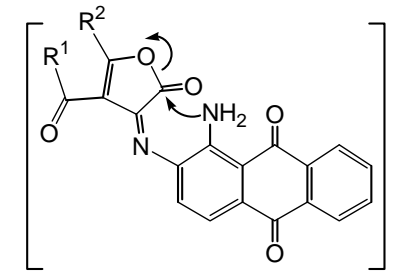

$$
1 d \leftrightarrow 3 d
$$

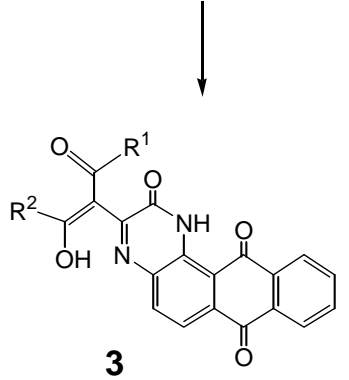

$$
\begin{aligned}
& \text { 1, 3: } \mathbf{a} R^{1}, R^{2}=\mathrm{Ph} ; \mathbf{b} R^{1}, R^{2}=4-\mathrm{MeOPh} ; \mathbf{c} R^{1}, R^{2}=4-\mathrm{MePh} ; \\
& \quad \mathbf{d} R^{1}=\mathrm{Me}, \mathrm{R}^{2}=4-\mathrm{MePh} \text { e } R^{1}, \mathrm{R}^{2}=3,4-(\mathrm{MeO})_{2} \mathrm{Ph} ; \mathbf{f} \mathrm{R}^{1}=\mathrm{Ph}, \mathrm{R}^{2}=\mathrm{Ph}-\mathrm{CH}=\mathrm{CH}-
\end{aligned}
$$

Reaction conditions: (ii) Benzene, reflux, $2 \mathrm{~h}$.

The structures of $\mathbf{2}$ and $\mathbf{3}$ were confirmed by spectroscopic data and agree with those found for similar compounds $[4,9,14,15]$. In the NMR spectra, the methine proton signal (low intensity) also revealed that compound $\mathbf{2}$ occurs as tautomers $(\mathbf{2 A}$ and $\mathbf{2 B})$, with tautomer $\mathbf{2 B}$ as a minor contributor in DMSO- $d_{6}$ solutions (Scheme 3). The ${ }^{13} \mathrm{C}-\mathrm{NMR}$ spectroscopic data of $\mathbf{2}$ also agree with the proposed tautomeric structures.

Scheme 3. Tautomeric forms (2A and 2B) of 2a-e in DMSO- $d_{6}$.<smiles>[R]C(NC(=O)O)=C(C(=O)O)C(=O)O</smiles>

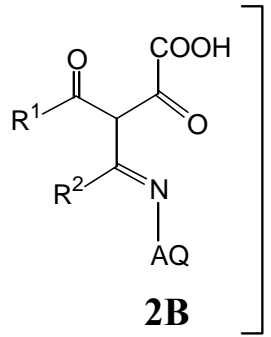


There was indication of tautomeric forms $\mathbf{3 A}$ (and 3B as a minor contributor) in 3a,f (but not $\mathbf{3 b}, \mathbf{c}, \mathbf{e}$ ) in their ${ }^{1} \mathrm{H}-\mathrm{NMR}$ spectra in DMSO- $d_{6}$ solution (Scheme 4). However, there was no signal for the methine proton belonging to the tautomer $3 \mathrm{C}$ in DMSO- $d_{6}$ solution. The ${ }^{13} \mathrm{C}-\mathrm{NMR}$ spectra of $\mathbf{3}$ could not be recorded due to its very low solubility in organic solvents, but the condensation was verified by the detection of the $\left[\mathrm{MH}^{+}\right]$and $\left[\mathrm{MH}^{+}-\mathrm{H}_{2} \mathrm{O}\right]$ signals.

Scheme 4. Tautomeric forms (3A-C) of 3a,f in DMSO- $d_{6}$.

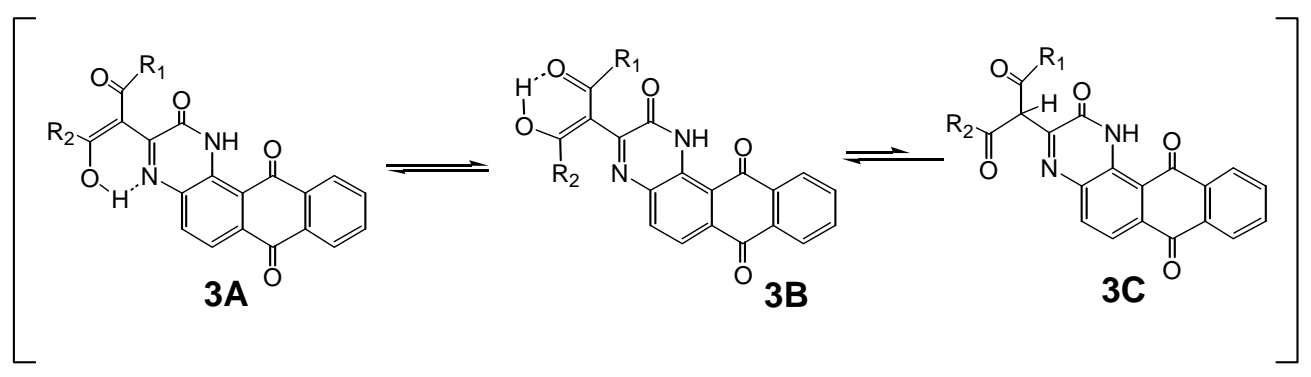

The UV-vis absorption spectra of 3a-c,e in DMF at a concentration of $3.333 \times 10^{-5} \mathrm{~mol} / \mathrm{L}$ are shown in Figure 2. The results are also listed in Table 1. In the visible region, the molar absorption coefficients and the absorption maxima of compounds 3 were observed in the range $1.119 \times 10^{4}$ to $1.545 \times 10^{4} \mathrm{dm}^{3} \mathrm{~mol}^{-1} \mathrm{~cm}^{-1}$ (380-394 nm), $1.161 \times 10^{4}$ to $1.557 \times 10^{4}(397-412 \mathrm{~nm}), 1.467 \times 10^{4}$ to $1.842 \times 10^{4}$ $(498-515 \mathrm{~nm})$, there being no significant difference between them. The visible absorption spectrum is dominated by the characteristic anthraquinone bands in the region 380-400 $\mathrm{nm}$ [15].

Figure 2. UV-Vis spectra of compound 3 in DMF.

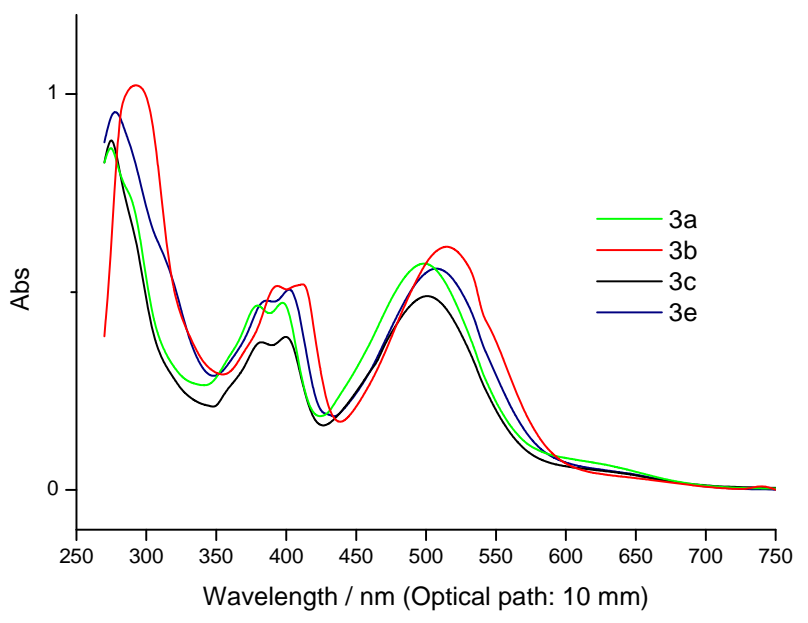


Table 1: UV-Vis spectral data of 3.

\begin{tabular}{|c|c|c|c|c|}
\hline \multirow{3}{*}{$\begin{array}{c}\text { Compounds } \\
3 a \\
\end{array}$} & \multicolumn{4}{|c|}{ UV-Vis } \\
\hline & \multicolumn{4}{|c|}{$\begin{array}{c}\lambda_{1-4 \max }(\mathrm{nm}), \\
\varepsilon_{1-4}\left(\text { liter } \mathrm{mol}^{-1} \mathrm{~cm}^{-1}\right)\end{array}$} \\
\hline & $\begin{array}{c}274, \\
2.592 \times 10^{4}\end{array}$ & $\begin{array}{c}380 \\
1.398 \times 10^{4}\end{array}$ & $\begin{array}{c}397 \\
1.416 \times 10^{4}\end{array}$ & $\begin{array}{c}498 \\
1.716 \times 10^{4}\end{array}$ \\
\hline $3 b$ & $\begin{array}{c}293 \\
3.066 \times 10^{4}\end{array}$ & $\begin{array}{c}394, \\
1.545 \times 10^{4}\end{array}$ & $\begin{array}{c}412 \\
1.557 \times 10^{4}\end{array}$ & $\begin{array}{c}515, \\
1.842 \times 10^{4}\end{array}$ \\
\hline $3 c$ & $\begin{array}{c}275 \\
2.652 \times 10^{4}\end{array}$ & $\begin{array}{c}382 \\
1.119 \times 10^{4}\end{array}$ & $\begin{array}{c}400 \\
1.161 \times 10^{4}\end{array}$ & $\begin{array}{c}501 \\
1.467 \times 10^{4}\end{array}$ \\
\hline $3 e$ & $\begin{array}{c}278 \\
2.865 \times 10^{4}\end{array}$ & $\begin{array}{c}386 \\
1.434 \times 10^{4}\end{array}$ & $\begin{array}{c}402, \\
1.518 \times 10^{4}\end{array}$ & $\begin{array}{c}507, \\
1.677 \times 10^{4}\end{array}$ \\
\hline
\end{tabular}

\section{Experimental Section}

\subsection{General}

Solvents were purchased from Merck and Carlo Erba. Diaminoanthra-9,10-quinones were purchased from Aldrich and used without further purification. ${ }^{1} \mathrm{H}$ - and ${ }^{13} \mathrm{C}-\mathrm{NMR}$ spectra were recorded using a Bruker Ultrashield spectrometer operating at $300.13 \mathrm{MHz}\left({ }^{1} \mathrm{H}\right)$ and $75.47 \mathrm{MHz}\left({ }^{13} \mathrm{C}\right)$. UV-vis spectra: Shimadzu UV-1280 spectrophotometer. IR Spectra: Jasco Plus Model 460 FTIR spectrometer; in $\mathrm{cm}^{-1}$. Elemental analyses $(\mathrm{C}, \mathrm{H}, \mathrm{N})$ were performed using a Leco CHNS-O 932. Melting points were measured with an Electrothermal 9200 apparatus. GC/MS measurements were performed using a gas chromatograph (Model 6890 Series)-mass selective detector (Model 5973N) system (Agilent Technologies).

\subsection{General Procedure for the Preparation of Compounds 2a-e}

Compound $1(1 \mathrm{mmol})$ and 1,2-/1,4-DAAQ $(1 \mathrm{mmol})$ in benzene $(60 \mathrm{~mL})$ was stirred for $48 \mathrm{~h}$. The precipitate 2 was filtered off and recrystallized from DMF.

4-[(4-Amino-9,10-dioxo-9,10-dihydroanthracen-1-yl)amino]-3-benzoyl-2-oxo-4-phenylbut-3-enoic acid (2a): Obtained from 1a $(0.278 \mathrm{~g}, 1 \mathrm{mmol})$ and 1,4-DAAQ $(0.238 \mathrm{~g}, 1 \mathrm{mmol})$. Mp. $195-196{ }^{\circ} \mathrm{C}$ as dark-blue crystals $(0.415 \mathrm{~g}, 80 \%)$; ${ }^{1} \mathrm{H}-\mathrm{NMR}\left(\mathrm{DMSO}-\mathrm{d}_{6}\right): \delta=13.50(1 \mathrm{H}, \mathrm{s}, \mathrm{NH}), 8.77-6.35(16 \mathrm{H}, \mathrm{m}$, aromatic), $7.16\left(2 \mathrm{H}, \mathrm{s}, \mathrm{NH}_{2}\right.$, br), 4.89 (Ar-CO-CH-CO, tautomer $\mathbf{B}$ ), $\mathrm{COOH}$ not observed; ${ }^{13} \mathrm{C}-\mathrm{NMR}$ $\left(\right.$ DMSO- $\left.d_{6}\right): \delta=193.29,192.53,188.81,184.79,183.47,181.49(\mathrm{C}=\mathrm{O}), 157.60,152.96,150.81$, $147.19,139.82,137.98,135.72,135.04,134.82,134.55,134.12,134.05,133.95,133.84,133.71$, $133.47,133.08,132.68,132.20,132.07,130.92,130.47,129.98,129.70,129.57,129.40,129.29$, 129.12 , 129.04, 128.86, 128.69, 128.15, 128.00, 127.87, 127.06, 126.46, 126.20, 124.43, 120.96, 118.32, 113.17, 111.94, 110.63, 110.20, 107.97, 93.69 (C=C, arom., aliph.), 64.05 (-CH-); IR (KBr) v 
$3450\left(\mathrm{OH}\right.$, br); 3445, $3427\left(\mathrm{NH}_{2}\right) ; 3302(\mathrm{NH}) ; 1724,1690,1670,1636 \mathrm{~cm}^{-1}(\mathrm{C}=\mathrm{O})$; Anal. Calcd for $\mathrm{C}_{31} \mathrm{H}_{20} \mathrm{~N}_{2} \mathrm{O}_{6}$ (516.5): C, 72.09; H, 3.90; N, 5.42. Found: C, 72.26; H, 3.88; N, 5.28.

4-[(4-Amino-9,10-dioxo-9,10-dihydroanthracen-1-yl)amino]-3-(4-methoxybenzoyl)-4-(4-methoxyphenyl)-2-oxobut-3-enoic acid (2b): Obtained from $\mathbf{1 b}(0.338 \mathrm{~g}, 1 \mathrm{mmol})$ and 1,4-DAAQ (0.238 g, 1 mmol). Mp 215-216 ${ }^{\circ} \mathrm{C}$ as black crystals $(0.503 \mathrm{~g}, 87 \%) ;{ }^{1} \mathrm{H}-\mathrm{NMR}$ (DMSO-d $\left.d_{6}\right): \delta=13.55,13.48(1 \mathrm{H}$, d, NH), 8.97-6.70 (14H, m, aromatic), 7.07 (2H, s, NH , br), 4.72 (Ar-CO-CH-CO, tautomer B), 3.86, $3.79\left(6 \mathrm{H}, \mathrm{s}, \mathrm{OCH}_{3}\right), \mathrm{COOH}$ not observed; ${ }^{13} \mathrm{C}-\mathrm{NMR}$ (DMSO- $\left.d_{6}\right): \delta=192.73,191.76,191.50,184.62$, 183.51, $181.49(\mathrm{C}=\mathrm{O}, \mathrm{C}=\mathrm{N}), 164.44,163.45,162.77,161.41\left(\mathrm{C}=\mathrm{C}-\mathrm{OCH}_{3}\right.$, tautomer $\mathbf{A}$ and $\left.\mathbf{B}\right), 157.78$, $147.20,135.07,134.56,134.12,133.98,133.08,132.70,131.79,131.72,131.36,131.04,129.91$, 129.29, 128.72, 127.59, 127.08, 126.47, 126.21, 114.77, 114.56, 113.54, 107.97, 91.94 (C=C, arom., aliph.), 65.24 (-CH-) 36.24, 34.64, $31.23\left(\mathrm{OCH}_{3}\right)$; IR (KBr) v $3455\left(\mathrm{OH}\right.$, br); $3425\left(\mathrm{NH}_{2}\right)$; $3300(\mathrm{NH})$; 1726, 1686, 1664, $1638 \mathrm{~cm}^{-1}(\mathrm{C}=\mathrm{O})$; Anal. Calcd for $\mathrm{C}_{33} \mathrm{H}_{24} \mathrm{~N}_{2} \mathrm{O}_{8}$ (576.6): C, 68.75; H, 4.20; N, 4.86. Found: C, 68.84; H, 4.40; N, 4.81 .

4-[(4-Amino-9,10-dioxo-9,10-dihydroanthracen-1-yl)amino]-3-(4-methylbenzoyl)-4-(4-methylphenyl)2-oxobut-3-enoic acid (2c): Obtained from $1 \mathrm{c}(0.306 \mathrm{~g}, 1 \mathrm{mmol})$ and 1,4-DAAQ (0.238 g, $1 \mathrm{mmol})$. Mp $220-221^{\circ} \mathrm{C}$ as navy-blue crystals $(0.493 \mathrm{~g}, 90 \%) ;{ }^{1} \mathrm{H}-\mathrm{NMR}$ (DMSO- $\left.d_{6}\right): \delta=13.54,13.49(1 \mathrm{H}, \mathrm{d}$, $\mathrm{NH}), 8.77-6.62$ (14H, m, aromatic), $7.58\left(2 \mathrm{H}, \mathrm{s}, \mathrm{NH}_{2}\right.$, br), 4.79 (Ar-CO-CH-CO, tautomer B), 3.45 $(1 \mathrm{H}, \mathrm{s}, \mathrm{COOH}, \mathrm{br}), 2.41,2.31\left(14 \mathrm{H}, \mathrm{s}, \mathrm{CH}_{3}\right) ;{ }^{13} \mathrm{C}-\mathrm{NMR}\left(\mathrm{DMSO}-d_{6}\right): \delta=192.95,192.69,192.61$, $187.08,183.55,181.49(\mathrm{C}=\mathrm{O}, \mathrm{C}=\mathrm{N}), 157.69,150.24,147.21,145.48,135.11,134.56,134.00,133.90$, $133.33,133.15,132.70,130.99,130.09,129.87,129.79,129.46,129.38,129.30,128.80,128.17$, 127.88, 127.18, 127.11, 126.49, 126.21, 118.36, 110.22, 107.95 (C=C, arom., alph.), 63.83 (-CH-), 21.71, $21.55\left(\mathrm{CH}_{3}\right)$; IR $(\mathrm{KBr})$ v $3480\left(\mathrm{OH}\right.$, br); $3442\left(\mathrm{NH}_{2}\right) ; 3308(\mathrm{NH}) ; 1725,1689,1671,1663,1638$ $\mathrm{cm}^{-1}(\mathrm{C}=\mathrm{O})$; Anal. Calcd for $\mathrm{C}_{33} \mathrm{H}_{24} \mathrm{~N}_{2} \mathrm{O}_{6}$ (544.6): C, 72.78; H, 4.44; N, 5.14. Found: C, 72.92; H, 4.35; N, 4.96 .

3-Acetyl-4-[(4-amino-9,10-dioxo-9,10-dihydroanthracen-1-yl)amino]-4-(4-methylphenyl)-2-oxobut-3enoic acid (2d): Obtained from 1d (0.230 g, $1 \mathrm{mmol})$ and 1,4-DAAQ (0.238 g, $1 \mathrm{mmol})$. Mp 174-175 ${ }^{\circ} \mathrm{C}$ as brown crystals $(0.419 \mathrm{~g}, 90 \%) ;{ }^{1} \mathrm{H}-\mathrm{NMR}$ (DMSO- $\left.d_{6}\right): \delta=13.54,13.44(1 \mathrm{H}, \mathrm{d}, \mathrm{NH}), 8.92-6.86$ $\left(10 \mathrm{H}, \mathrm{m}\right.$, aromatic), $7.58\left(2 \mathrm{H}, \mathrm{s}, \mathrm{NH}_{2}, \mathrm{br}\right), 4.64$ (Ar-CO-CH-CO, tautomer B), $3.51(1 \mathrm{H}, \mathrm{s}, \mathrm{COOH}, \mathrm{br})$, $2.39\left(3 \mathrm{H}, \mathrm{s}, \mathrm{COCH}_{3}\right), 2.22\left(3 \mathrm{H}, \mathrm{s}, \mathrm{C}=\mathrm{C}-\mathrm{CH}_{3}\right) ;{ }^{13} \mathrm{C}-\mathrm{NMR}\left(\mathrm{DMSO}-d_{6}\right): \delta=200.05,192.45,186.70$, 183.39, $181.47(\mathrm{C}=\mathrm{O}, \mathrm{C}=\mathrm{N}), 152.97,150.05,147.19,146.02,136.21,135.72,134.92,134.54,133.92$, $133.76,133.09,132.69,131.21,130.20,130.04,129.88,129.70,129.37,129.27,128.07,127.00$, 126.41, 126.20, 117.89, 116.76, 109.96, 107.95, 105.96 (C=C, arom., alph.), $36.25\left(\mathrm{COCH}_{3}\right), 21.59$ $\left(\mathrm{CH}_{3}\right)$; IR (KBr) v $3475(\mathrm{OH}, \mathrm{br}) ; 3432\left(\mathrm{NH}_{2}\right) ; 3300(\mathrm{NH}) ; 1734,1685,1666,1637,1638 \mathrm{~cm}^{-1}(\mathrm{C}=\mathrm{O})$; Anal. Calcd for $\mathrm{C}_{27} \mathrm{H}_{20} \mathrm{~N}_{2} \mathrm{O}_{6}$ (468.4): C, 69.22; H, 4.30; N, 5.98. Found: C, 69.16; H, 4.15; N, 5.75.

4-[(1-Amino-9,10-dioxo-9,10-dihydroanthracen-2-yl)amino]-3-benzoyl-2-oxo-4-phenylbut-3-enoic acid (2e): Obtained from 1a $(0.278 \mathrm{~g}, 1 \mathrm{mmol})$ and 1,2-DAAQ $(0.238 \mathrm{~g}, 1 \mathrm{mmol})$. Mp $255-256{ }^{\circ} \mathrm{C}$ as brown crystals $(0.386 \mathrm{~g}, 75 \%)$; ${ }^{1} \mathrm{H}-\mathrm{NMR}\left(\mathrm{DMSO}-d_{6}\right): \delta=15.23(1 \mathrm{H}, \mathrm{s}, \mathrm{NH}), 8.31-6.94(16 \mathrm{H}, \mathrm{m}$, aromatic), $7.16\left(2 \mathrm{H}, \mathrm{s}, \mathrm{NH}_{2}, \mathrm{br}\right), 3.38(1 \mathrm{H}, \mathrm{s}, \mathrm{br}, \mathrm{COOH}) ;{ }^{13} \mathrm{C}-\mathrm{NMR}\left(\mathrm{DMSO}-d_{6}\right): \delta=194.27,191.87$, 
188.77, 186.80, 185.32, $181.44(\mathrm{C}=\mathrm{O}, \mathrm{C}=\mathrm{N}), 144.54,142.51,142.14,134,56,134.29,133.54,131,07$, $129.63,129.15,128.43,128.10,127.86,127.66,127.51,127.02,126.60,126.41,125.63,124.56$, 124.18, 116.88, 109.01 (C=C, arom., alph.); IR (KBr) v $3435\left(\mathrm{OH}, \mathrm{br}, \mathrm{NH}_{2}\right) ; 3211(\mathrm{NH}) ; 1717,1658$, 1649, $1639 \mathrm{~cm}^{-1}(\mathrm{C}=\mathrm{O})$; Anal. Calcd. for $\mathrm{C}_{31} \mathrm{H}_{20} \mathrm{~N}_{2} \mathrm{O}_{6}$ (516.5): C, 72.09; H, 3.90; N, 5.42\%. Found: C, 72.39; H, 3.72; N, 5.61.

\subsection{General Procedure for the Preparation of Compounds 3a-c,e,f}

Compound 1 ( $1 \mathrm{mmol})$ and 1,2-DAAQ $(1 \mathrm{mmol})$ in benzene $(40 \mathrm{ml})$ was refluxed for $2 \mathrm{~h}$. The redcoloured precipitate $(\mathbf{3})$ was filtered off and recrystallized from DMSO.

3-(1-Benzoyl-2-oxo-2-phenylethyl)naphtho[2,3-f]quinoxaline-2,7,12(1H)-trione (3a): Obtained from 1a $(0.278 \mathrm{~g}, 1 \mathrm{mmol})$ and 1,2-DAAQ (0.238 g, $1 \mathrm{mmol})$. Mp 341-343 ${ }^{\circ} \mathrm{C} ; 0.517 \mathrm{~g}, 94 \%$; ${ }^{1} \mathrm{H}-\mathrm{NMR}$ $\left(\mathrm{DMSO}-d_{6}\right): \delta=15.70,15.07(1 \mathrm{H}, \mathrm{s}, \mathrm{OH}, \mathrm{br}), 13.25,12.27(1 \mathrm{H}, \mathrm{s}, \mathrm{NH}-\mathrm{C}=\mathrm{O}, \mathrm{br}), 8.33-6.79(16 \mathrm{H}, \mathrm{m}$, $\mathrm{Ar}-\mathrm{H})$; IR (KBr) v $3435(\mathrm{OH}), 3210(\mathrm{NH}), 3072,2925(\mathrm{C}-\mathrm{H}), 1694,1661,1651,1621 \mathrm{~cm}^{-1}(\mathrm{C}=\mathrm{O})$; MS $(\mathrm{ESI})^{+}: \mathrm{m} / \mathrm{z} 498(1 \%)[\mathrm{M}+\mathrm{H}]^{+}$; Anal. Calcd for $\mathrm{C}_{31} \mathrm{H}_{18} \mathrm{~N}_{2} \mathrm{O}_{5}$ (498.5): C, 74.69; H, 3.64; N, 5.62. Found: C, 74.78; H, 3.68; N, 5.60 .

3-[1-(4-Methoxybenzoyl)-2-(4-methoxyphenyl)-2-oxoethyl]naphtho[2,3-f]quinoxaline-2,7,12(1H)trione (3b): Obtained from $\mathbf{1 b}(0.338 \mathrm{~g}, 1 \mathrm{mmol})$ and 1,2-DAAQ $(0.238 \mathrm{~g}, 1 \mathrm{mmol})$; Mp 347-349 ${ }^{\circ} \mathrm{C}$; $0.517 \mathrm{~g}, 92 \% ;{ }^{1} \mathrm{H}-\mathrm{NMR}\left(\mathrm{DMSO}-d_{6}\right): \delta=15.45(1 \mathrm{H}, \mathrm{s}, \mathrm{OH}, \mathrm{br}), 12.45(1 \mathrm{H}, \mathrm{s}, \mathrm{NHCO}, \mathrm{br}), 8.48-6.70$ $(14 \mathrm{H}, \mathrm{m}, \mathrm{Ar}-\mathrm{H}), 3.80,3.74\left(6 \mathrm{H}, \mathrm{s}, \mathrm{OCH}_{3}\right)$; IR $(\mathrm{KBr}) \vee 3437(\mathrm{OH}), 3214(\mathrm{NH}), 3069,2926(\mathrm{C}-\mathrm{H})$, 1695, 1664, 1654, $1638 \mathrm{~cm}^{-1}(\mathrm{C}=\mathrm{O})$; MS (ESI) ${ }^{+}: \mathrm{m} / \mathrm{z} 559(5 \%)[\mathrm{M}+\mathrm{H}]^{+}, \mathrm{m} / \mathrm{z} 541(6 \%)\left[\mathrm{MH}-\mathrm{H}_{2} \mathrm{O}\right]^{+}$; Anal. Calcd for $\mathrm{C}_{33} \mathrm{H}_{22} \mathrm{~N}_{2} \mathrm{O}_{7}$ (558.5): C, 70.96; H, 3.97; N, 5.02. Found: C, 70.79; H, 3.93; N, 5.17.

3-[1-(4-Methylbenzoyl)-2-(4-methylphenyl)-2-oxoethyl] naphtho[2,3-f] quinoxaline-2,7,12(1H)-trione

(3c): Obtained from 1c $(0.306 \mathrm{~g}, 1 \mathrm{mmol})$ and 1,2-DAAQ $(0.238 \mathrm{~g}, 1 \mathrm{mmol})$; $\mathrm{Mp} \mathrm{315-317}{ }^{\circ} \mathrm{C} ; 0.473 \mathrm{~g}$, 90\%; ${ }^{1} \mathrm{H}-\mathrm{NMR}$ (DMSO-d $)$ ): $\delta=15.50(1 \mathrm{H}, \mathrm{s}, \mathrm{OH}, \mathrm{br}), 12.43(1 \mathrm{H}, \mathrm{s}, \mathrm{NHCO}), 8.29-7.06(14 \mathrm{H}, \mathrm{m}, \mathrm{Ar}-$ H), 2.34, $2.26\left(6 \mathrm{H}, \mathrm{s}, \mathrm{CH}_{3}\right)$; IR ( $\left.\mathrm{KBr}\right) \vee 3432(\mathrm{OH}), 3200(\mathrm{NH}), 3071,2914(\mathrm{C}-\mathrm{H}), 1693,1663,1656$, $1638 \mathrm{~cm}^{-1}(\mathrm{C}=\mathrm{O})$; MS (ESI) $)^{+}: \mathrm{m} / \mathrm{z} 526(13 \%)[\mathrm{M}+\mathrm{H}]^{+}, \mathrm{m} / \mathrm{z} 525(25 \%)[\mathrm{M}]^{+}$; Anal. Calcd for $\mathrm{C}_{33} \mathrm{H}_{22} \mathrm{~N}_{2} \mathrm{O}_{5}$ (526.5): C, 75.28; H, 4.21; N, 5.32. Found: C, 75.04; H, 4.29; N, 5.49.

3-[1-(3,4-Dimethoxybenzoyl)-2-(3,4-dimethoxyphenyl)-2-oxoethyl]naphtho[2,3-f]quinoxaline-

2,7,12(1H)-trione (3e): Obtained from $1 \mathrm{e}(0.398 \mathrm{~g}, 1 \mathrm{mmol})$ and 1,2-DAAQ $(0.238 \mathrm{~g}, 1 \mathrm{mmol})$; Mp 309-311 ${ }^{\circ} \mathrm{C}$; $0.587 \mathrm{~g}, 95 \%$; ${ }^{1} \mathrm{H}-\mathrm{NMR}$ (DMSO-d $\left.{ }_{6}\right): \delta=15.44(1 \mathrm{H}, \mathrm{s}, \mathrm{OH}, \mathrm{br}), 12.35(1 \mathrm{H}, \mathrm{s}, \mathrm{NHCO})$, 8.33-6.88 (12H, m, Ar-H), 3.81, 3.79, 3.75, $3.48\left(12 \mathrm{H}, \mathrm{s}, \mathrm{OCH}_{3}\right)$; IR (KBr) v $3436(\mathrm{OH}), 3253(\mathrm{NH})$, 3075, $2932(\mathrm{C}-\mathrm{H}), 1698,1660,1654,1640 \mathrm{~cm}^{-1}(\mathrm{C}=\mathrm{O})$; MS (ESI) ${ }^{+}: \mathrm{m} / \mathrm{z} 619(6 \%)[\mathrm{M}+\mathrm{H}]^{+}, \mathrm{m} / \mathrm{z} 601$ (2\%) $\left[\mathrm{MH}-\mathrm{H}_{2} \mathrm{O}\right]^{+}$; Anal. Calcd for $\mathrm{C}_{35} \mathrm{H}_{26} \mathrm{~N}_{2} \mathrm{O}_{9}$ (618.6): C, 67.96; H, 4.24; N, 4.53. Found C, 68.17; H, $4.24 ; \mathrm{N}, 4.60$.

3-[(3E)-1-Benzoyl-2-oxo-4-phenylbut-3-en-1-yl] naphtho[2,3-f]quinoxaline-2,7,12(1H)-trione (3f): Obtained from 1 (f (0.304 g, $1 \mathrm{mmol})$ and 1,2-DAAQ (0.238 g, $1 \mathrm{mmol})$; Mp 307-308 ${ }^{\circ} \mathrm{C} ; 0.472 \mathrm{~g}, 90 \%$; 
${ }^{1} \mathrm{H}-\mathrm{NMR}\left(\mathrm{DMSO}-d_{6}\right): \delta=15.64,14.55(1 \mathrm{H}, \mathrm{s}, \mathrm{OH}, \mathrm{br}), 12.35,12.11(1 \mathrm{H}, \mathrm{s}, \mathrm{NH}), 8.28-6.82(16 \mathrm{H}, \mathrm{m}$, Ar-H), $7.74(1 \mathrm{H}, \mathrm{d}, \mathrm{J}=15.4 \mathrm{~Hz}, \mathrm{CH}=), 6.64(1 \mathrm{H}, \mathrm{d}, \mathrm{J}=15.3 \mathrm{~Hz}, \mathrm{CH}=)$; IR $(\mathrm{KBr}) v 3435(\mathrm{OH}), 3213$ $(\mathrm{NH}), 3062,2853(\mathrm{C}-\mathrm{H}), 1696,1664,1657,1638 \mathrm{~cm}^{-1}(\mathrm{C}=\mathrm{O}) ; \mathrm{MS}(\mathrm{ESI})^{+}: \mathrm{m} / \mathrm{z} 525(6 \%)[\mathrm{M}+\mathrm{H}]^{+}, \mathrm{m} / \mathrm{z}$ $508(3 \%)\left[\mathrm{MH}-\mathrm{H}_{2} \mathrm{O}\right]^{+}$; Anal. Calcd for $\mathrm{C}_{33} \mathrm{H}_{20} \mathrm{~N}_{2} \mathrm{O}_{5}$ (524.5): C, 75.56; H, 3.84; N, 5.34. Found: C, $75.53 ; \mathrm{H}, 3.80 ; \mathrm{N}, 5.41$.

\section{Conclusions}

We have designed and easily synthesized novel naphtho[2,3-f]quinoxaline-2,7,12(1H)-triones and anthra-9,10-quinones in good to excellent yields as potential vat dyes from furan-2,3-diones. Their spectroscopic properties in solution and in the solid state are reported. For commercial dye production, reactions with high yields and relatively straightforward chemistry are preferred. It was seen that furan-2,3-diones have good reactivity to meet these expectations. We believe that preparation of various heterocyclic dyes based on furan-2,3-dione chemistry might make a contribution to the development of high performance pigments. Performances of new dyes will be tested in future studies.

\section{Acknowledgements}

The author is very grateful to Dr. İrfan Koca and Ress. Assist. Bilal Nişancı for technical assistance.

\section{References and Notes}

1. Thetford, D.; Chorlton, A.P. Investigation of vat dyes as potential high performance pigments. Dyes Pigments 2004, 61, 49-62.

2. Zhang, Y.; Hou, W.; Tan, Y. Structure and dyeing properties of some anthraquinone violet acid dyes. Dyes Pigments 1996, 34, 25-35.

3. Orban, N.; Boldizsar, I.; Szucs, Z.; Danos, B. Influence of different elicitors on the synthesis of anthraquinone derivatives in Rubia tinctorum L. cell suspension cultures. Dyes Pigments 2007, 77, 249-257, and references therein.

4. Matsui, M.; Taniguchi, S.; Suzuki, M.; Wang, M.; Funabiki, K.; Shiozaki, H. Dyes produced by the reaction of 1,2,3,4-tetrafluoro-9,10-anthraquinones with bifunctional nucleophiles. Dyes Pigments 2005, 65, 211-220.

5. Kollenz, G.; Heilmayer, W. Furan-2,3-diones-versatile synthons in heterocyclic chemistry. Trends Heterocycl. Chem. 1993, 3, 379-395.

6. Üngören, Ş.H.; Saçmacı, M.; Akçamur, Y.; Arıcı, C.; Ülkü, D. Synthesis and characterization of new $\alpha, \beta$-unsaturated $\gamma$-lactones: Alkyl 3-acetyl-4-hydroxy-2-methyl-5-oxo-2,5-dihydrofuranylcarbamates. J. Heterocycl. Chem. 2005, 42, 685-689.

7. Şener, A.; Akbaş, E.; Şener, M.K. Synthesis and some reactions of 4-benzoyl-5-phenyl-1-pyridin2-yl-1H-pyrazole-3-carboxylic acid. Turk. J. Chem. 2004, 28, 271-277.

8. Akbaş, E.; Aslanoğlu, F. Syntheses of some new 1H-pyrazole, pyridazin-3(2H)-one, and oxazin4-one derivatives. Heteroatom Chem. 2006, 17, 8-12. 
9. Amer, A.; Ventura, M.; Zimmer, H. Substituted $\gamma$-lactones. XXX (1). Reactions of $\alpha$-keto- $\beta$ substituted- $\gamma$-lactones with diamines. J. Heterocycl. Chem. 1983, 20, 359-364.

10. Ziegler, E.; Eder, M.; Belegratis, C.; Prewedourakis, E. Synthesen von heterocyclen, 103. mitt.: Über reaktionen mit oxalylchlorid. Monatsh. Chem. 1967, 98, 2249-2251.

11. Hökelek, T.; Sarıpınar. E.; Yıldırım. İ.; Akkurt. M.; Akçamur. Y. 4-(4-Methoxybenzoyl)-5-(4methoxyphenyl)-2,3-dihydro-2,3-furandione. Acta Cryst. E 2002, 58, 30-32.

12. Yıldırım, İ.; Koca, İ. Synthesis, characterization and some reactions of novel 4-aroyl-5-aryl-2,3dihydro-2,3-furandiones. Kuwait J. Sci. Eng. 2005, 32, 49-60.

13. Üngören, Ş.H.; Deniz, B.; Altural, B. Synthesis of novel 2,3-dihydro-2,3-furandione and $1 \mathrm{H}-$ pyrazole-3-carboxylic acid derivatives. Asian J. Chem. 2004; 16, 805-810.

14. Kappe, C.O.; Terpetschnig, E.; Penn, G.; Kollenz, G.; Peters, K.; Peters, E.M.; von Schnering, H.G. Reactions of cyclic oxalyl compounds .37. substituent effects on the site of nucleophilicattack at 1H-pyrrole-2,3-diones. Liebigs Ann. 1995, 37, 537-543.

15. Lopez, R.; Boys, D.; Loeb, B.; Zuloaga, F. Synthesis, crystal structure, molecular orbital calculations and electronic properties of 2,3-di(2-pyridyl)naphtho[2,3-f]quinoxaline-7,12quinone(Aqdpp). J. Chem. Soc., Perkin Trans. 2 1998, 877-883.

Sample Availability: Samples of the compounds $\mathbf{2 a - e}$ and $\mathbf{3 a , b , c , e , f}$ are available from the authors.

(C) 2009 by the authors; licensee Molecular Diversity Preservation International, Basel, Switzerland. This article is an open-access article distributed under the terms and conditions of the Creative Commons Attribution license (http://creativecommons.org/licenses/by/3.0/). 